\title{
MONITORING OF DILATION CRACKS AND VIBRATIONS CAUSED BY BLASTING WORKS AT THE QUARRY OF MOKRA ON SURROUNDING CONSTRUCTION OBJECTS
}

The quarry of Mokra, whose locality comprises administrative area of three communities, has cooperated with our Institute for a longer time, communicating with the authorities and people, as regards environmental safety and quality of living in the area. Monitoring of the conditions by continual measurements of dilation cracking and blasting works seismic effects has been an indispensable part of the collaboration.

The measurement equipment was developed especially for the needs and condition of the quarry of Mokra, but can be used universally. This paper reports on technicalities of its development and experience of using it in practice.

Key words: seismic effect, dilation, vibration, blasting works, sensors, data file.

\section{Introduction}

Blasting works necessarily imply collateral damage of different rate. This is influenced by many factors - local geological condition, weather, technical parameters of blasting works, but also by the quality of the buildings affected. From the side of general public, they are seen as detrimental by all means, although they can be within authorized limits of standards.

Considering the fact that also in future blasting will stay to be a principle method of rock extraction, and consequent collateral damage cannot be excluded but only regulated, an existence of a quality system of damage measurement is of major importance. Reliable data provided on the matter will definitely be concurrent to all arising problems and possible remedying action - instant or prospective.

\section{Continuous Measurement of Dilation Cracks and Vibrations}

\subsection{Measurement System Requirements and Measurement Principle}

As already mentioned, quarry blasting works can have adverse seismic effects on buildings in their vicinity. These seismic effects decrease exponentially with the increasing distance of objects from the blasting action. The stability of construction objects can exceed limits of tolerance when physical magnitudes of related parameters get over defined standards. Cracking is the initial indication of a building static damage. The cracks can be caused by static or dynamic forces. Affecting building foundations, unstable subsoil static forces can cause cracking in absence of any seismic load. The seismic load tolerance limits on construction objects are defined by the State Standards. Apart from subsoil instability, also temperature changes can influence cracking.

To be able to differentiate seismic dynamic loads caused by blasting from other sources of dynamic or static loads on buildings, it is necessary to develop a specific measurement system that would continually and concurrently monitor visually identifiable and measureable dilation cracks caused by dynamic forces (tremors) of blasting works.

Measurement and evaluation units:

Dilation gauge, Vibration gauge, Gauge service circuits, Timing circuits, Signal transmission modules, Long service power-supply unit, Initiating programme module, Microprocessor SW for measurement and control circuits, Data computing gear, Reliable protection of equipment inclusive its fixing

\section{Dilation measurements}

Enclosed in a waterproof housing, two dilatometers are installed at each measurement point.

Basic operation requirements:

- Uncertainty of measurement $\quad 5 \%$

- Maximum measurement range $15 \mathrm{~mm}$

\footnotetext{
* Milan Mikolas ${ }^{1}$, Roman Donocik ${ }^{2}$, Jana Bartonova ${ }^{3}$, Michal Vanek ${ }^{3}$, Martin Mikolas ${ }^{4}$

${ }^{1}$ Institute of Mining Engineering and Safety, Faculty of Mining and Geology, VSB - TU Ostrava, Czech Republic, E-mail: milan.mikolas@vsb.cz

${ }^{2}$ Ceskomoravsky cement, a.s., nastupnicka spolecnost, MOKRA-HORAKOV, Czech Republic

${ }^{3}$ Institute of Economics and Management Systems, Faculty of Mining and Geology, VSB - TU Ostrava, Czech Republic

${ }^{4}$ Institue of Applied informatics, Faculty of Economics, VSB-TU Ostrava, Czech Republic
} 
- Working temperature from

- Sensor attachment spacing

$-10{ }^{\circ} \mathrm{C}$

$200 \mathrm{~mm}$

Individual sensors are attached by 2 ball joints. The sensors are perpendicular to each other. They both traverse the dilation crack measured. The dilation outcome value is calculated by vector analysis so that the results reflect the linear shift perpendicular to the dilation tangent and concurrent linear movement at the same point. The outcome file dilation values are provided in units and tenths of millimetres. The sensors are connected to the measurement unit by an electrical cable.

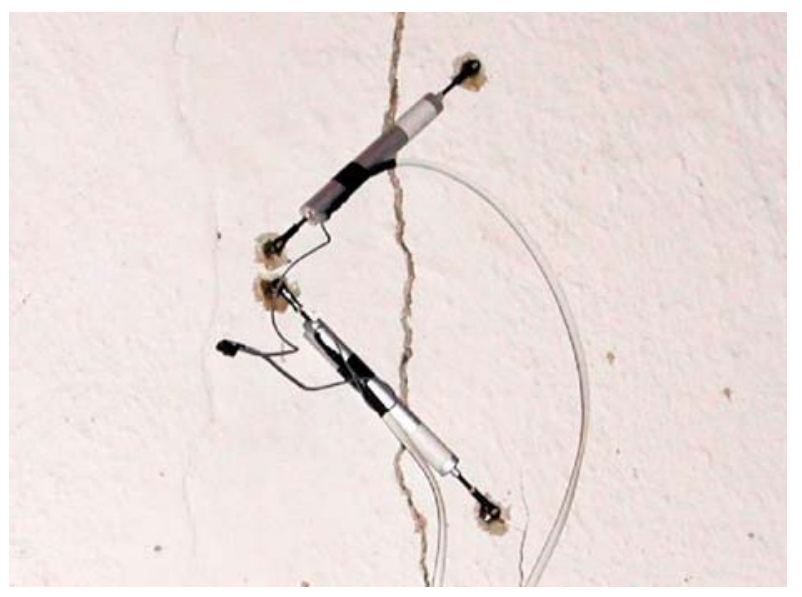

Fig. 1 Installation of dilatometers at measurement point

\section{Vibration gauge}

The vibration gauges are placed in a box that is permanently fixed next to the dilation sensors. The sensors are vertically and horizontally oriented respectively. The vibration gauges are constituted by two piezoelectric accelerometers. Nevertheless, the required parameter for seismic effect evaluation of blasting works on construction buildings is the speed of vibration. As such the piezoelectric accelerometers must be completed by integration circuits that can transfer with satisfactory accuracy the values of mechanical acceleration to values of vibration speeds. The levels of electrical input values for integrators must be amplified.

Basic operational requirements:

- Uncertainty of vibration speed measurements $5 \%$

- Frequency range $5 \%$

- Operational temperature from $-10{ }^{\circ} \mathrm{C}$

As ambient air-pressure changes could adversely affect the vibration readings, a solid mechanical attachment of the vibrometer body to the measured medium must be provided for.

The simplest method of vibration acceleration measurement is to put a load, $m$, on a spring of characteristics, $k$. The spring oscillation displacement is $x$. It is valid that
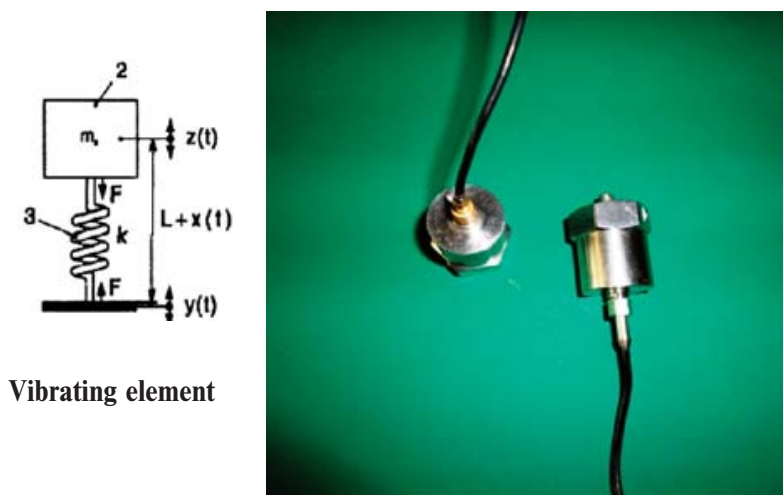

Where: 2 Solid body with mass, $\mathrm{m}$

3 Spring with elasticity constant, $\mathrm{k}$

$F \quad$ Spring force, $\mathrm{F}$, affecting the solid body, 2

$L$ Distance between the solid body surface and central mass point $z(t)$ Position change with time,t

$y(t)$ Change of the position of the vibrating body

$x(t)$ Change of distance, $\mathrm{L}$, with time, $\mathrm{t}$

\section{Fig. 2 Vibrometers}

$$
F=k \cdot x,
$$

$F$ - Force vector [N],

$k$ - Spring characteristics $\left[\mathrm{Nm}^{-1}\right]$

$x$ - Vector of magnitude (and direction) of the spring mass point [m]

\section{Calibration of sensors}

The accelerometers had been calibrated by a standard vibrometer before being put in their casing box. Before their assembly in situ, the dilatometers, inclusive the reference voltage source had been calibrated by a standard slide calliper:

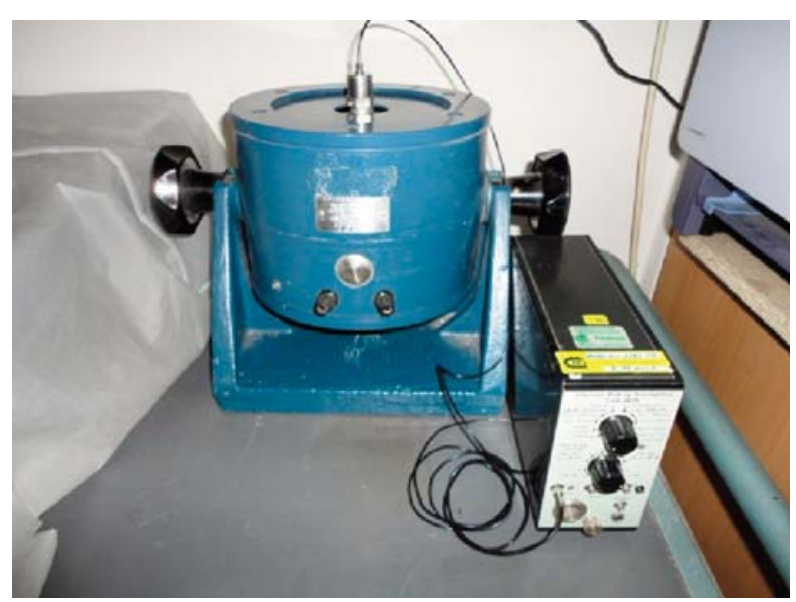

Fig. 3 Standard Vibrometer's Calibration 


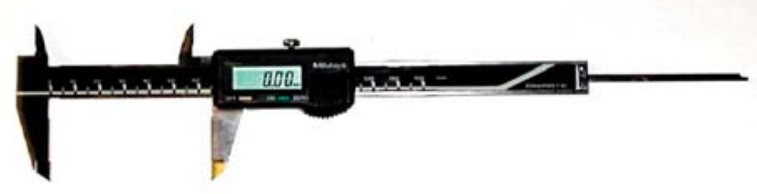

Fig. 4 Secondary Length Standard Micrometer for Calibration of Sensors

\section{Measurement Unit Block Chart}

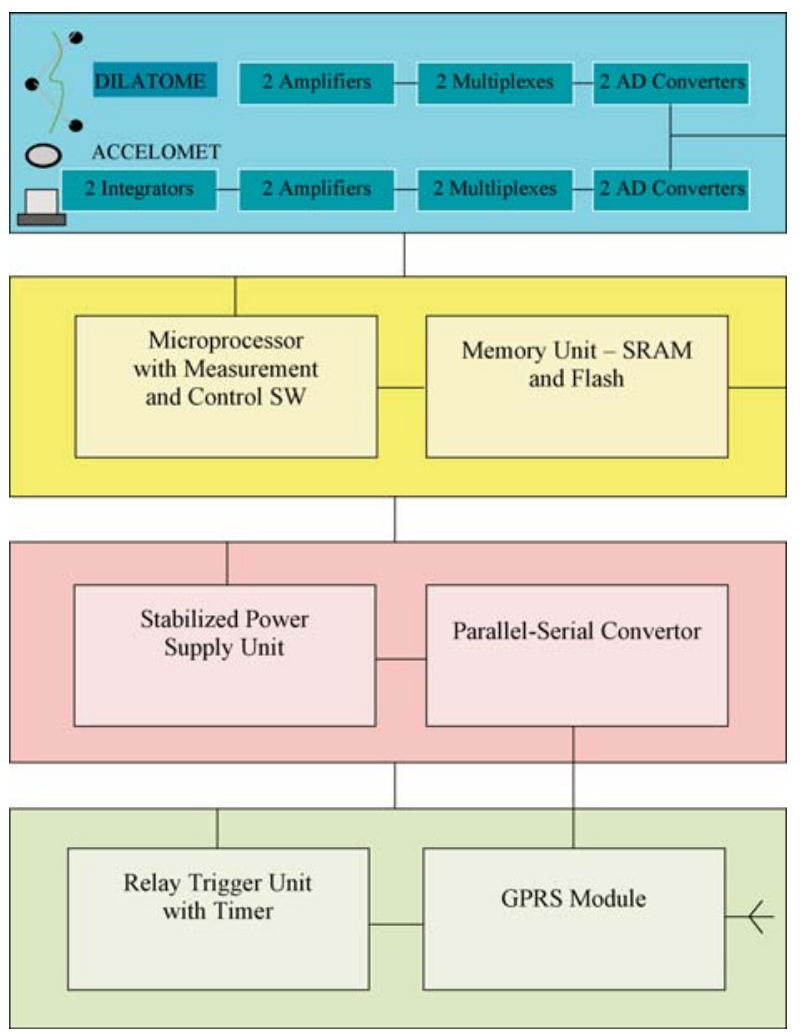

\section{Measurement Site}

In the photograph, we can see the installation of a measurement unit at a specific site. The positioning of sensors vis-à-vis the monitored crack is clearly illustrated. As already mentioned, the accelerometers are encased in the box, and as such, the box must be firmly attached to the wall so that no distortion of original information occurs. The best attaching technique is to cement the box onto the wall. As continuous measurement assumes long term monitoring action, also weather conditions must be taken into account as well as human factor influences. For example, the measurement installation could be unintentionally damaged during maintenance works at the site.

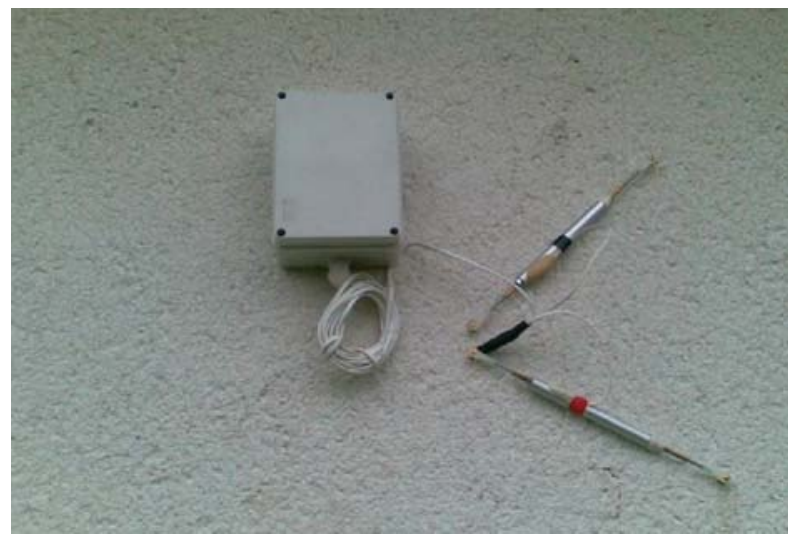

Fig. 5 Installation of a measurement unit

Another limiting factor of the proper installation can be also posed by options for possible wireless transmission of monitoring data. The quality of installation and good wireless transmission of measurement signals can decisively affect reliability and accuracy of the monitored data. The measurement site should satisfactorily represent typical condition of the monitored building object, facilitating objective assessment of the seismic effect of blasting works on the building monitored. Such prerequisites ask for a thorough on-the-spot inspection and identification of the place, in which dilation cracking may occur.

\section{Measurement and Assessment Circuits}

All functions of the measurement system are controlled by an 8-Bit industrial microprocessor. This chip, apart from the classical x 80 core, must comprise four pieces of 12-Bit approximation analogue-to-digital convertors with amplifiers, also minimally three pieces of 8-Bit input/output ports, a serial UART circuit, a precise clock oscillator of the computer (no high tact time speeds are required, rather low current power feed, approx. tenths of $\mathrm{mA}$, is more important). The processor should work at a feed power of $3 \mathrm{~V}$.

The input ports receive signals from the dilation and tremor sensors for twelve hours, namely between $6 \mathrm{am}$ and $6 \mathrm{pm}$ every day. The signals are processed and maximum values of each 12-Hour measurement cycle, inclusive relevant timings, are recorded and transmitted in the form of a SMS to the receiver unit at night hours (cellular phone with an output for PC). The SMS transmitter is active only a few seconds daily so that power consumption is kept low.

Low feed power values enable long-term operation of the monitoring units (in fact several years if alkaline batteries are used).

The timing function is provided by a relay switch unit. The relay unit is controlled by the processor working on command of a specific programme. All timing data and measurement intervals are managed by a circuit of an accurate real time counter. A microprocessor input port receives the timing data needed. 
An important part of the tremor sensors (vibrometers) is an integrating high input impedance charge amplifier. It converts vibration movement acceleration values to their related speeds. The mechanical vibration speeds are of decisive importance concerning monitoring and assessment of damage of building objects. If maximum vibration speeds exceed standard values, initial slight damage may occur.

For that reason, final output data of monitoring are regularly watched and seismic effect of blasting works is officially assessed.

Movement can be recorded:

$$
\begin{aligned}
& s(t) s(t)=\int v(t) d t \\
& v(t) v(t)=\int a(t) d t v(t)=d s(t) / d t \\
& a(t) a(t) a(t)=d v(t) / d t
\end{aligned}
$$

Where: $s(t)$ Distance with respect to time, $t[\mathrm{~m}]$; $v(t)$ Velocity with respect to time, $t[\mathrm{~m} / \mathrm{s}]$; $a(t)$ Acceleration with respect to time, $t\left[\mathrm{~m} / \mathrm{s}^{2}\right]$; $t$ Time.

Transfers for harmonious signals (based on acceleration):

$$
\begin{aligned}
& a(t)=A \sin (\omega t) \\
& v(t)=(-A / \omega) \cos (\omega t) \\
& s(t)=\left(=A / \omega^{2}\right) \sin (\omega t)
\end{aligned}
$$

Where: $a(t)$ Acceleration with respect to time, $t\left[\mathrm{~m} / \mathrm{s}^{2}\right]$;

$v(t)$ Velocity with respect to time, $t[\mathrm{~m} / \mathrm{s}]$;

$s(t)$ Distance with respect to time, $t[\mathrm{~m}]$;

$A$ Amplitude of oscillation displacement;

$\omega$ Circular frequency;

$t$ Time

The measurements of vibration by accelerometers provide for the starting point of the whole monitoring process. The accelerometers are the most common sensors of largely different design. If damage effect of vibrations is monitored, the vibration speed - the so called vibration magnitude - is the best criterion. It enables usage of acceleration sensors with the following integration and aggregation. A direct usage of speed sensors is also functionally viable.

\section{Dilation in Buildings}

No-load effects, like volume changes due to changing temperature and humidity, rheological effects, and shape modification of foundations joints cause mechanical tensions in buildings that can many times exceed normal vertical load stresses or horizontal stresses of wind or multidirectional seismic stresses caused by blasting works.
In order to reduce these detrimental effects, it is of advantage to sectionalize the building construction, dividing the whole construction in smaller parts of different tendency to shape changes and of different settlement rate. Individual building sections are connected by construction (dilation) joints.

The construction joints should be located at places of extreme load occurrence, abrupt change of construction rigidity, change of construction system/design, sudden change of load, change of height or overall structuring, also in places of geological faults or irregularities.

Structuring of buildings in smaller parts is a preventive action that reduces probability of damage. Designing of construction joints also depends on the specific structure and rigidity of the supporting system. It is necessary to analyze effects of load, temperature, humidity, shrinking, shape modification and change of the foundation joint.

The temperature effect is of cyclic nature and changes volume and shape of buildings that many times behave like a beam, which expands or shrinks with changing temperature. If the temperature changes differ along the whole length of the construction unit, a characteristic deformation occurs - it bends. If humidity increases, porous materials start deforming; this may also mean increasing of their length. Effects of humidity are usually more pronounced than those of temperature.

The material shrinkage concerns volume changes due to humidity and the material inner structure.

The design of construction joints concerns primarily points of maximum strain or stress, like the points: Maximum pressure load, Maximum strain stress, Maximum shear stress.

\section{Data Transmission}

A 12-Bit approximation A/D convertor changes the dilation sensor values from digital to analogue form. The digitization is made to an accuracy of 0.01 millimetres. In fact, such high accuracy is not needed and after the vector analyses perpendicular to and along the dilation crack has been made, the final values are adjusted to sign sequences of the order, 0.1 millimetres before the subsequent conversion.

The outputs of the accelerometers are integrated by an analogue circuit to related vibration speeds. The seismic curve maps are not purely harmonious but of stochastic character, which implies an increase of uncertainty of mechanical oscillation measurement up to $5 \%$. Nevertheless, a statistical analysis of the final data file excludes this uncertainty, and it cannot influence the ultimate assessment of the seismic effects of blasting works performed.

The signal sampling is $1 \mathrm{msec}$. This fully conforms to oscillation maps measured. After digitization, maximum amplitude values 
of both sensors are chosen and they are converted to sign sequences of the order of $0.1 \mathrm{~mm} / \mathrm{sec}$.

Along with the dilation and maximum speed values, also part of the final numerical sign sequences are date and time of blasting works related to the seismic maximum. Assisting digital data, concerning identity of the specific measurement unit and the state of its batteries, are also included in the information sequence.

The data file numerical form is transferred from the microprocessor serial output (UART) to the transmission module (GPRS) for SMS communication, which follows at a pre-set night hour.

A common cellular phone equipped with an output connector for USB cable media date transmission to PC serves the purpose of the receiver.

This is an example of the SMS digital sign sequence of a specific measurement unit:

\section{2}

The sequence represents values in the following order:

17 Horizontal component maximum oscillation speed $v=1.7 \mathrm{~mm} / \mathrm{s}$

1525 Horizontal component time record

19 Vertical component maximum oscillation speed

15 H 25 MM

$v=1.9 \mathrm{~mm} / \mathrm{s}$

1412 Vertical component time record

$14 \mathrm{H} 12 \mathrm{MM}$

45 Dilatometer value perpendicular to cracking $4.5 \mathrm{~mL}$

56 Dilatometer value along the cracking $\quad 5.6 \mathrm{~mL}$

43 Power supply voltage 4.3V

22 Measurement unit serial number

\section{Data Processing}

The SMS communication from the receiver is deciphered to provide for a PC data file. An assessment programme serves the purpose of the final data processing. The SW decoding unit not only deciphers the input digital sequence, but also performs other statistical and calculation tasks that can assess the rate of seismic damage of buildings. Apart from that, also relative movement of the dilation crack is calculated from the daily information provision by the dilatometers. The dilation crack values are related to the initial level of 0.0 millimetre dilation.

The calculation and check-up results are entered into working table files. Each major anomaly is related to standard levels of tol- erance. If a standard tolerance is violated, the management of the quarry is immediately informed.

If dilation crack and vibration values are beyond limits of tolerance, a monthly monitoring report is produced giving maximum readings of all sites. A brief conclusive evaluation is also a part of the report. A continuous supervising of the batteries of all measurement units is another important part of the whole monitoring process (all measurement units have their own battery pack).

\section{Evaluation of the Monitoring Results}

To minimize seismic damage on buildings by quarry blasting works, there should be an instant feedback from the measurements units about the effects of the blasting technology used. Regular and systematic monitoring of blasting works seismic affects, which is based on measurements of the maximum vibration amplitudes, can authoritatively decide on the stability of the building objects seismically affected by blasting. The measurement feedback can influence options of the blasting works, concerning timing of individual blasts, their mass, explosive agent quality, and drilling schemes used. Local geological conditions are of major importance, as regards propagation of damaging seismic waves. Although, these conditions cannot be influenced, nevertheless, they must be accounted for in the overall assessment of the damage caused by blasting.

Authorized measurements of technical tremor caused by blasting works are precisely regulated by legal and metrological measures. The results of these measurements influence decisively the method of blasting works used or imply necessity of its change. The authorized measurements are performed in relatively long time periods (once or twice in a year) and hardly can account for all the aspects of the complex occurrence.

A continuous and permanent monitoring of blasting works effects (monitoring of dilation cracking and vibration magnitudes at specific sites of the buildings exposed) is needed. It is precise and can provide for conclusive evidence if authorities are faced to decide about complaints and claims of the proprietors of the buildings damaged. Only modern IT communication services, which work on wireless principle, and fast computer processing, can provide for instant feedback and conclusive objective evidence needed.

The article is based on seismic measurements of organizations Ceskomoravsky cement, a.s., nastupnicka spolecnost, Mokra and MET-ROCK, s.r.o. It is based on original measurement and therefore the article does not contain any references to literature.

\section{References}

[1] VOJTA, A.: Generalni projekt trhacich praci velkeho rozsahu a Technologicky postup trhacich praci maleho rozsahu $v$ dobyvacim prostoru Mokra [General Project for Major Blasting Works and Technology of Minor Blasting Works for the Field of Mining of Mokra], Mokra, 2002.

[2] CSN 7300 40: Zatizeni stavebnich objektu technickou seizmicitou a jejich odezva [Technical Seismicity Load on Buildings, Standard, CZ]. 